\title{
Modelos de entrenamiento en cirugía mínimamente invasiva para pieloplastia laparoscópica: revisión de la literatura*
}

\section{Training Models in Minimally Invasive Surgery for Laparoscopic Pyeloplasty: Review of the Literature}

Recepción: 30/01/2018 | Aceptación: 04/04/2018

\author{
Pedro Villamizar \\ Pontificia Universidad Javeriana, Colombia \\ Marco Ángel \\ Pontificia Universidad Javeriana, Colombia \\ Valentina Corchuelo \\ Pontificia Universidad Javeriana, Colombia \\ Miguel Delgado \\ Pontificia Universidad Javeriana, Colombia \\ STEPHANIE ORDÓÑEZ \\ Pontificia Universidad Javeriana, Colombia \\ Marcela Tejada \\ Pontificia Universidad Javeriana, Colombia \\ Freddy Moreno ${ }^{a}$ \\ Pontificia Universidad Javeriana, Colombia
}

\footnotetext{
a Correspondencia:

fmorenog@javerianacali.edu.co
}

Cómo citar: Villamizar P, Ángel M, Corchuelo V, Delgado M, Ordóñez S, Tejada M, Moreno F. Modelos de entrenamiento en cirugía mínimamente invasiva para pieloplastia laparoscópica: revisión de la literatura. Univ. Med. 2018;59(4). doi: https:// doi.org/10.11144/Javeriana.umed59-4.mecm

\section{RESUMEN}

Introducción: la pieloplastia laparoscópica es un procedimiento mínimamente invasivo empleado para solucionar quirúrgicamente estenosis, bloqueo u obstrucción de la unión ureteropélvica o pieloureteral. Objetivo: explorar los diferentes modelos de entrenamiento en cirugía mínimamente invasiva para pieloplastia laparoscópica que se encuentran reportados en la literatura. Materiales y métodos: se revisó la literatura con búsqueda sistemática en PubMed, Google Académico y SciELO, mediante la declaración PRISMA y la combinación de los descriptores médicos en salud Model training AND laparoscopic pyeloplasty. Además, se empleó GoPubMed para obtener información bibliométrica que contextualizara las redes de investigación y publicación entre los diferentes modelos de entrenamiento para pieloplastia laparoscópica. Resultados: Se incluyeron 17 referencias en la búsqueda sistemática de la literatura con los que se realizó la discusión a partir de la usabilidad, la estética y el tiempo operatorio de la pieloplastia laparoscópica. Conclusión: Todos los modelos de entrenamiento contribuyen a que los operadores disminuyan los tiempos quirúrgicos, alcanzando valores próximos a la pieloplastia laparoscópica in situ, inclusive, a la pieloplastia abierta. De forma particular, los modelos anatomopatológicos elaborados con modelamiento de siliconas logran un notable acercamiento a la realidad morfológica a partir de la estética de las estructuras y permiten aumentar el número de intentos quirúrgicos y la cantidad de horas de práctica. 
Palabras clave

cirugía; procedimientos quirúrgicos reconstructivos; laparoscopia; pelvis renal; uréter

\begin{abstract}
Introduction: Laparoscopic pyeloplasty is a minimally invasive procedure used to surgically correct stenosis, blockage or obstruction of the ureteropelvic junction or pyeloureteral junction. Objective: To explore the different models of training in minimally invasive surgery for laparoscopic pyeloplasty that are reported in the literature. Materials and methods: A literature review with systematic search was carried out in PubMed, Google Scholar and SciELO, through the PRISMA Declaration and the combination of the medical descriptors in health "Model training AND laparoscopic pyeloplasty". In addition, GoPubMed was used to obtain bibliometric information that contextualized the research and publication networks among the different training models for laparoscopic pyeloplasty. Results: We included 17 references in the systematic review of the literature with which the discussion was made based on the usability, aesthetics and operative time of laparoscopic pyeloplasty. Conclusion: All the training models contribute to those operators reduce surgical times, reaching values close to laparoscopic pyeloplasty in situ, including open pyeloplasty. In particular, anatomopathological models made with silicone modeling achieve a remarkable approach to the morphological reality from the aesthetics of the structures and allow increasing the number of surgical attempts and the number of hours of practice.

Keywords

surgery; reconstructive surgical procedures; laparoscopy; kidney pelvis; ureter
\end{abstract}

\section{Introducción}

La cirugía mínimamente invasiva (CMI) consiste en un conjunto de procedimientos quirúrgicos diagnósticos y terapéuticos que emplea diferentes vías morfofisiológicas o mínimos abordajes para introducir diferentes instrumentos y acceder así a determinada parte del cuerpo humano. Dentro de un concepto global, la CMI incluye las técnicas de endoscopia, cirugía laparoscópica y cirugía percutánea, las cuales son llevadas a cabo por casi todas las especialidades médico-quirúrgicas, y cuyo propósito incluye la disminución del estrés operatorio (reducción de las respuestas inflamatoria e inmunológica) y del riesgo quirúrgico (reducción al máximo de los efectos del trauma quirúrgico [dolor y sangrado]). Todo ello contribuye a una rápida recuperación e incorporación de los pacientes a su vida cotidiana, lo que finalmente reduce las estancias y las necesidades intrahospitalarias por simplificación de los cuidados postoperatorios. De esta forma, el éxito de la CMI radica en: 1) una correcta indicación a partir no solo de la vía de abordaje, sino también de la seguridad, la eficiencia y el costo frente a la cirugía convencional, y 2) en la experiencia y destreza del equipo de médicos cirujanos, quienes son en últimas los que garantizan el éxito de los resultados en términos de morbilidad y mortalidad $(1,2,3)$.

Uno de los procedimientos incluidos en la CMI es la cirugía laparoscópica, que consiste en una técnica quirúrgica que permite llevar a cabo intervenciones sin necesidad de un campo operatorio abierto. Una de las especializaciones médicas que más han desarrollado la técnica quirúrgica laparoscópica es la pediatría, en la cual los procedimientos quirúrgicos abiertos —considerados convencionales- se han remplazado por la cirugía laparoscópica urológica, dentro de la que se incluye la pieloplastia laparoscópica $(4,5)$.

Inicialmente, la pieloplastia desmembrada fue descrita, en 1949, por Anderson y Hynes (6) como la técnica quirúrgica abierta empleada para solucionar la obstrucción de la unión ureteropélvica (o pieloureteral) en adultos, quizás la localización más frecuente de las obstrucciones de las vías urinarias superiores. Esta alteración estructural y funcional impide el flujo anterógrado de la orina de la pelvis renal al uréter asociado — de forma intrínseca $(80 \%$ de los casos) - y disminuye el músculo liso en la capa muscular de la túnica mucosa, de tal forma que es remplazado por un tejido conectivo denso irregular aperistáltico que ocluye la luz del uréter e impide el paso de la orina. Otras de las causas etiopatológicas asociadas — de forma extrínseca (20\% de los casos) — son la inserción anormal del uréter proximal en la pelvis renal o la presencia de un vaso sanguíneo aberrante que comprime la unión pieloureteral. Si bien la mayoría de las obstrucciones son detectadas de forma temprana durante las ecografías prenatales (primarias o congénitas), estas mismas pueden manifestarse clínicamente en los adultos o también pueden 
presentarse de novo (secundarias o adquiridas) $(7,8,9)$.

En términos generales, el procedimiento quirúrgico consiste en la resección del sitio obstruido, la escisión del tejido fibrosado y la unión del uréter con la pelvis renal. De esta forma, la pieloplastia abierta se ha constituido en el procedimiento estándar para solucionar las obstrucciones pieloureterales con un éxito superior al 90\% $(10,11)$. Sin embargo, con el desarrollo de la cirugía laparoscópica, Schuessler et al. (12), en 1993, desarrollaron la pieloplastia desmembrada laparoscópica (reproduciendo la técnica de Anderson y Hynes) como una CMI para reconstruir la unión del uréter con la pelvis renal y solucionar todos los síntomas relacionados con la obstrucción, el dolor, la disfunción renal progresiva, la infección y la litiasis; además de, con un nivel éxito muy similar al procedimiento estándar (de hasta el 89\%), obtener los beneficios que ofrece per se la CMI $(13,14,15,16,17)$.

Debido a que la obstrucción de la unión ureteropélvica es la alteración congénita de mayor incidencia de las vías urinarias superiores (uno en 1500 nacidos vivos en un año) y que es la causa más frecuente de hidronefrosis asociada a daño renal progresivo $(18,19)$, resulta fundamental un diagnóstico y tratamiento oportuno, de tal forma que la intervención temprana condicione la recuperación de la función renal en menor o mayor grado; mientras que la intervención tardía solo podrá limitar de acuerdo con el tiempo de evolución- el daño existente $(20,21)$. Por ello, a partir del primer informe de Peters et al. (22), en 1995, hasta la actualidad, se ha podido evidenciar que pieloplastia laparoscópica ha alcanzado tasas de éxito comparables con las de la cirugía abierta y cuando las intervenciones se han realizado de forma temprana en el paciente pediátrico $(13,23,24,25)$. Sin embargo, para realizar la pieloplastia laparoscópica con una tasa de éxito alta y obtener los beneficios de la CMI, los médicos cirujanos deben demostrar sus habilidades quirúrgicas en el plano tridimensional mientras observan en un plano bidimensional, además de manipular los instrumentos y tejidos a una distancia mayor a 30 centímetros $(22,26,27,28)$.

Dado que la pieloplastia laparoscópica es un procedimiento quirúrgico técnicamente difícil asociado a la alta exigencia de la coordinación mano-ojo y a la precisión de la sutura y anudamiento intracorporal-, durante su residencia en cirugía pediátrica o en cirugía urológica los médicos deben capacitarse de forma repetitiva para desarrollar competencias y adquirir habilidades reconstructivas mediante técnicas laparoscópicas, antes de entrar al quirófano. Para ello se han implementado una serie de métodos (modelos de entrenamiento) que simulan las condiciones y circunstancias clínicas reales, como simuladores de realidad virtual que permiten una familiarización con el espacio-tiempo durante el abordaje quirúrgico, modelos de pelvis inanimadas confeccionadas en materiales poliméricos con texturas y densidades que ofrecen una resistencia similar a los tejidos orgánicos, laboratorios con cadáveres humanos y laboratorios con biomodelos animales que permiten la transferencia de habilidades a la realidad anatómica $(29,30,31,32)$. No obstante, en la literatura se han reportado pocos modelos patológicos costo-efectivos y éticos (artificiales y naturales) de pieloplastia, que permitan el entrenamiento de la pieloplastia laparoscopia para lograr la optimización de una técnica de sutura y anudamiento estandarizado en tiempos que resulten eficientes y de una cirugía reconstructiva segura y eficaz $(33,34,35)$.

Por tanto, el objetivo de esta revisión fue explorar los diferentes modelos de entrenamiento en cirugía mínimamente invasiva para pieloplastia laparoscópica que se encuentran reportados en la literatura.

\section{Materiales y métodos}

Se revisó la literatura sobre el tema con una búsqueda sistemática en PubMed, Google Académico y SciELO, mediante las pautas de la Declaración PRISMA y los descriptores médicos en salud Model training y laparoscopic pyeloplasty, combinados con el operador booleano AND 
(Model training AND laparoscopic pyeloplasty). Se incluyeron artículos que evaluaron un modelo de entrenamiento para pieloplastia laparoscópica en idioma inglés (figura 1). Se empleó GoPubMed, a través de la combinación de las palabras clave Model training AND laparoscopic pyeloplasty para obtener información bibliométrica que contextualizara las redes de investigación entre los diferentes modelos de entrenamiento para pieloplastia laparoscópica a través del año y el país publicación, además de las revistas que más han publicado sobre la temática.

\section{Figura 1}

Búsqueda de referencias de acuerdo con la metodología PRISMA (36)

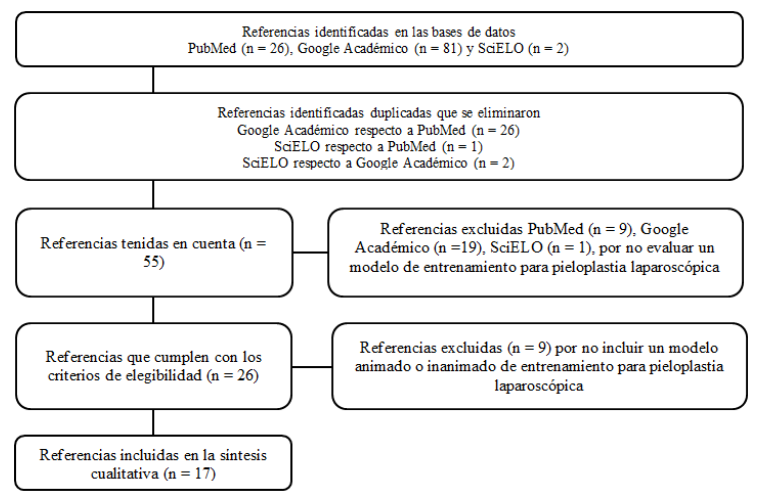

\section{Resultados}

De acuerdo con la metodología PRISMA (36) se obtuvieron, luego de los filtros iniciales, 55 referencias, de las cuales se excluyeron 29 , porque no cumplieron con el criterio de inclusión a partir de la lectura del título y el resumen. De las 26 referencias resultantes fueron eliminadas 9 , por no incluir modelos animados (tejidos orgánicos obtenidos de biomodelos animales) o inanimados (elementos realizados en materiales poliméricos). Finalmente, se incluyeron 17 referencias con las que se realizó la discusión a partir de la usabilidad, la estética y el tiempo operatorio (anexo).

$\mathrm{Al}$ respecto, de los 17 artículos, 16 correspondieron a estudios que compararon los diferentes modelos de entrenamiento y uno fue un estudio comparativo entre la pieloplastia abierta y la pieloplastia laparoscópica. Todos los estudios se llevaron a cabo en los últimos 17 años. Las publicaciones que trabajaron modelos animales consistieron en 7 estudios en riñones de cerdo, 4 en diferentes órganos de pollo (esófago, intestinos, buche y piel) y uno en órganos de cerdo (uréter e intestinos), lo cual correspondió al 70,6\%; mientras que las publicaciones que trabajaron en modelos secos consistieron en 3 riñones modelados en silicona, un guante de látex y un riñón plastinado, lo cual correspondió al 29,4\%. Dentro de los operadores, se destacan 5 estudios con residentes de urología, 5 con cirujanos urólogos, 4 con cirujanos con experiencia en cirugía laparoscópica, 2 con cirujanos sin experiencia y uno con fellow de urología pediátrica.

Finalmente, se pudo obtener una visión general de la tendencia global de interés de publicación de estudios que describieron y compararon los diferentes modelos de entrenamiento para pieloplastia laparoscópica, y se encontró que se han publicado 26 artículos, de los cuales 4 tienen como país de origen de la revista a Estados Unidos; de allí, el resto de publicaciones se distribuyen en Europa, Asia y Australia. Todos estos artículos se han publicado fundamentalmente en revistas de urología, sobresaliendo el Journal of Endourology, revista publicada por la Sociedad de Endourología, la cual tiene un índice $\mathrm{H}$ de SCImago Elsevier ${ }^{\circledR}$ de 78 y con el cual se ubica en el cuartil Q1, un factor de impacto de Thompson Reuters ${ }^{\circledR}$ de 2,27 y un índice H5 de 35 en Google Scholar. En esta revista fueron publicados ocho artículos tenidos en cuenta en esta revisión sistemática $(32,35,36,37,38,39,40,41,42,43,44,45,46,47,48$, $49,50,51)$.

\section{Discusión}

Debido a que la pieloplastia laparoscópica es un procedimiento quirúrgico que exige ciertas competencias y habilidades a los cirujanos urólogos y pediatras, se requiere en los diferentes programas de formación que se completen una cantidad de horas de entrenamiento mediante diferentes modelos de simulación. Al revisar la literatura, se encuentran diferentes métodos de entrenamiento que incluyen modelos 
biológicos y modelos artificiales (denominados secos), los cuales han sido evaluados por diferentes investigadores con el propósito de encontrar el modelo que ofrezca mejor usabilidad (aproximación a la realidad operatoria), mejor estética (aproximación al aspecto del modelo anatomopatológico) y mejor tiempo operatorio (aproximación a los tiempos de pieloplastia abierta como gold standard). Por ello, el método más común de evaluación de los modelos de entrenamiento en cirugía mínimamente invasiva para pieloplastia laparoscópica resulta de la disposición del modelo (riñón con obstrucción de la unión pieloureteral) en una caja "pélvica" de entrenamiento, al cual se accede a través de los diferentes instrumentos, de tal forma que se "simulan" las condiciones técnicas del procedimiento quirúrgico.

\section{Usabilidad}

Cada uno de los artículos manifestó que los modelos evaluados son usables y cumplen con las condiciones para desarrollar competencias y adquirir habilidades reconstructivas que ameriten sutura intracorpórea mediante técnicas laparoscópicas. De hecho, Zhang et al. (32) sugirieron un programa de entrenamiento multimodal que incluya diferentes modelos.

\section{Estética}

Una de las principales críticas que se les ha hecho a los modelos animales de obstrucción de la unión pieloureteral constituidos por diferentes órganos (diferentes al riñón y al uréter) y a los modelos secos, como el guante o las bombas de látex, es la falta de realismo con los componentes anatómicos in situ. Si bien el operador es consciente de que se encuentra en un ambiente de simulación, el mayor acercamiento que se pueda obtener a la realidad anatómica (estructura morfológica, texturas y variaciones anatomopatológicas) redunda en una mejor práctica. En este sentido, el riñón de cerdo resulta un modelo de sustituto pélvico renal adecuado y óptimo $(37,40,41,45,48,50)$. Sin embargo, las nuevas técnicas de modelamiento en silicona de diferentes órganos (incluido el riñón) con gran estética morfológica y funcional han permitido desarrollar modelos secos en los que la práctica de simulación sea bastante satisfactoria en términos de "sensación de realismo" al momento de la manipulación quirúrgica $(38,39,49)$.

\section{Tiempo operatorio}

En el momento de tener en cuenta la pieloplastia abierta como gold standard frente a la pieloplastia laparóscópica, uno de los factores de mayor incidencia es el tiempo quirúrgico. De esta forma, una de las indicaciones del uso de ambientes de simulación mediante el uso modelos de entrenamiento en cirugía mínimamente invasiva para pieloplastia laparoscópica es la reducción de los tiempos operatorios, específicamente al realizar la sutura y hacer el anudamiento intracorpóreo. En los diferentes modelos reportados en la literatura, y conforme aumenta el número de horas de práctica, el tiempo operatorio disminuye hasta alcanzar rendimientos óptimos comparados con la pieloplastia laparoscopia in situ, e inclusive comparado con la pieloplastia abierta, mucho más evidentes en el modelo animal de riñón de cerdo incorporado en la caja de entrenamiento pélvico $(35,40,41,42,43,44,46,47,50,51)$.

\section{Conclusiones}

De acuerdo con los artículos incluidos en esta revisión de la literatura, se concluye que los modelos de entrenamiento en CMI reproducen la complejidad técnica de la pieloplastia laparoscópica, lo cual contribuye a que los diferentes cirujanos especialistas desarrollen competencias y adquieran habilidades durante la reconstrucción de la unión pieloureteral mediante sutura y anudamiento intracorpóreo. Todos los modelos de entrenamiento ayudan a que los operadores disminuyan los tiempos quirúrgicos, alcanzando valores próximos a la pieloplastia laparoscópica in situ, incluso a la pieloplastia abierta. 
De esta forma, los modelos animales de riñón de cerdo logran una mayor aproximación a la realidad, pero no representan las condiciones anatomopatológicas de la obstrucción de la unión pieloureteral con otras estructuras anatómicas. De igual forma, los modelos de diferentes órganos de pollo si bien no cumplen los requisitos anteriores, se constituyen en simuladores de bajo costo y con cierta aproximación a la textura de los tejidos orgánicos. Finalmente, los modelos anatomopatológicos elaborados con siliconas logran un notable acercamiento a la realidad morfológica a partir de la estética de las estructuras, y aunque con un costo más elevado, la posibilidad de tener órganos y estructuras intercambiables aumenta el número de intentos quirúrgicos y la cantidad de horas de práctica, razón por la cual podrían ser considerados los modelos más costoefectivos durante los ejercicios de simulación.

\section{Conflictos de interés}

Los autores del artículo hacen constar que no existe, de manera directa o indirecta, ningún tipo de conflicto de interés que pueda poner en peligro la validez de lo comunicado.

\section{Referencias}

1. Vittimberga Jr FJ, Foley DP, Meyers WC, Callery MP. Laparoscopic surgery and the systemic immune response. Ann Surg. 1998;227(3):326-34.

2. Ochsner JL. Minimally invasive surgical procedures. Ochsner J. 2000;2(3):135-6.

3. Lera JM. Reflexiones sobre el pasado, presente y futuro de la cirugía mínimamente Invasive. An Sist Sanit Navar. 2005;28(Supl 3):7-10.
4. Castillo O, Cortés O. Complicaciones en cirugía laparoscópica urológica. Actas Urol Esp. 2006;30(5):541-54.

5. Belibasakis I, Kolostoumpis G, Makrygiannaki K. Current trends in minimally invasive reconstructive urology. J Robotic Surg. 2012;6(3):179-87.

6. Anderson JC, Hynes W. Retrocaval ureter: A case diagnosed preoperatively and treated successfully by a plastic operation. $\mathrm{Br} \mathrm{J}$ Urol. 1949;21(3):209-14.

7. Marcovich R, Jacobson AI, Aldana JP, Lee BR, Smith AD. Practice trends in contemporary management of adult ureteropelvic junction obstruction. Urology. 2003;62 (1):22-5.

8. Escobar F, Arbeláez S, Correa JJ, Gaviria F, Bonilla A, Ramírez LF. Experiencia en pieloplastia laparoscópica. Rev Urol Colomb. 2006;15(1):79-84.

9. Eden CG. Minimally invasive treatment of ureteropelvic junction obstruction: A critical analysis of results. Eur Urol. 2007;52 (4):983-9.

10. Valdivia JB, Sánchez MA, Sánchez M. Pieloplastia laparoscópica. Arch Esp Urol. 2001;55(6):679-86.

11. Fernández A, Carreño J, González R, Menéndez A, Albelo Y, Feder O. Pieloplastia laparoscópica: Experiencia inicial. Revista Cubana de Urología [Internet]. 2012;1(1):84-94. Disponible en: http://www.revurologia. sld.cu/index.php/rcu/article/view/10/1 3

12. Schuessler WW, Grune MT, Tecuanhuey LV, Preminger GM. Laparoscopic dismembered pyeloplasty. J Urol. 1993;150:1795-9.

13. Brooks JD, Kavoussi LR, Preminger GM, Schuessler WW, Moore RG. Comparison of open and endourologic approaches to the obstructed ureteropelvic junction. Urology. 1995;46(6):791-5. 
14. O'Reilly PH, Brooman PJ, Mak S, Jones M, Pickup C, Atkinson $\mathrm{C}$ et al. The long-term results of Anderson-Hynes pyeloplasty. BJU Int. 2001;87(4):287-9.

15. Moon DA, El Shazly MA, Chang CM, Gianduzzo TR, Eden CG. Laparoscopic pyeloplasty: Evolution of a new gold standard. Urology. 2006;67(5):932-6.

16. Rassweiler JJ, Subotic S, FeistSchwenk M, Sugiono M, Schulze M, Teber D, et al. Minimally invasive treatment of ureteropelvic junction obstruction: Long-term experience with an algorithm for laser endopyelotomy and laparoscopic retroperitoneal pyeloplasty. J Urol. 2007;177(3):1000-5.

17. El-Shazly MA, Moon DA, Eden CG. Laparoscopic pyeloplasty: Status and review of literature. J Endourol. 2007;21(7):673-8.

18. Johnston JH, Evans JP, Glassberg KI, Shapiro SR. Pelvic hydronephrosis in children: A review of 219 personal cases. J Urol. 1977;117(1):97-101.

19. Koff SA. Pathophysiology of ureteropelvic junction obstruction: Clinical and experimental observations. Urol Clin North Am. 1990;17(2):263-72.

20. Sánchez D, López J, Arocena J, Sanz G, Díez F, Rosell D, et al. Estenosis de la unión pieloureteral: Exposición de nuestra experiencia y revisión de la literatura. Actas Urol Esp. 2000;24(5):367-74.

21. Landa S, Maldonado W, Hernández G, Zaldiva J, Zepeda JT, Velázquez. Obstrucción pieloureteral: Revisión de 175 casos. Urología Pediátrica. 2002;17(1):32-8.

22. Peters CA, Schlussel RN, Retik AB. Paediatric laparoscopic dismembered pyeloplasty. J Urol. 1995;153(6):1962-5.

23. Meretyk I, Meretyk S, Clayman RV. Endopyelotomy: Comparison of ureteroscopic retrograde and antegrade percutaneous techniques. J Urol. 1992;148(3):775-82.

24. Tan HL. Laparoscopic AndersenHynes dismembered pyeloplasty in children. J Urol. 1999;162(3 Pt 2):1045-7.

25. Casale P, Grady RW, Joyner BD, Zeltser IS, Figueroa TE, Mitchell ME. Comparison of dismembered and nondismembered laparoscopic pyeloplasty in the pediatric patient. J Endourol. 2004;18(9):875-8.

26. Reddy M, Nerli RB, Bashetty R, Ravish IR. Laparoscopic dismembered pyeloplasty in children. J Urol. 2005;174(2):700-2.

27. Sweeney DD, Smaldone MC, Docimo SG. Minimally invasive surgery for urologic disease in children. Nat Clin Pract Urol. 2007;4(1):26-38.

28. Turrà $F$, Escolino $M$, Farina A, Settimi A, Esposito C, Varlet F. Pyeloplasty techniques using minimally invasive surgery (MIS) in pediatric patients. Transl Pediatr. 2016;5(4):251-5.

29. Keeley FX Jr, Eden CG, Tolley DA, Joyce AD. The British Association of Urological Surgeons: Guidelines for training in laparoscopy. BJU Int. 2007;100(2):379-81.

30. Aggarwal R, Moorthy K, Darzi A. Laparoscopic skills training and assessment. $\mathrm{Br} \mathrm{J}$ Surg. 2004;91(12):1549-58.

31. Fried GM, Feldman LS, Vassiliou $\mathrm{MC}$, Fraser SA, Stanbridge D, Ghitulescu G, et al. Proving the value of simulation in laparoscopic surgery. Ann Surg. 2004;240(3):518-28. 
32. Zhang $\mathrm{X}$, Zhang GX, Wang BJ, Ma X, Fu B, Shi TP et al. A multimodal training program for laparoscopic pyeloplasty. J Endourol. 2009;23(2):307-11.

33. Scott DJ, Bergen PC, Rege RV, Laycock R, Tesfay ST, Valentine RJ, et al. Laparoscopic training on bench models: better and more cost effective than operating room experience? J Am Coll Surg. 2000;191(3):272-83.

34. Scott DJ, Young WN, Tesfay ST, Frawley WH, Rege RV, Jones DB. Laparoscopic skills training. Am J Surg. 2001;182(2):137-42.

35. Ramachandran A, Kurien A, Patil P, Symons S, Ganpule A, Muthu V et al. A novel training model for laparoscopic pyeloplasty using chicken crop. J Endourol. 2008;22(4):725-8.

36. Liberati A, Altman DG, Tetzlaff J, Mulrow C, Gøtzsche PC, Ioannidis JP. The PRISMA statement for reporting systematic reviews and meta-analyses of studies that evaluate health care interventions: Explanation and elaboration. PLoS Med. 2009;6(7):e1000100.

37. Cicione A, Autorino R, Laguna MP, De Sio M, Micali S, Turna $B$ et al. Threedimensional technology facilitates surgical performance of novice laparoscopy surgeons: A quantitative assessment on a porcine kidney model. Urology. 2015;85(6):1252-6.

38. Cheung CL, Looi T, Lendvay TS, Drake JM, Farhat WA. Use of 3-dimensional printing technology and silicone modeling in surgical simulation: Development and face validation in pediatric laparoscopic pyeloplasty. J Surg Educ. 2014;71(5):762-7.

39. Poniatowski LH, Wolf JS Jr, Nakada

SY, Reihsen TE, Sainfort F, Sweet RM.
Validity and acceptability of a highfidelity physical simulation model for training of laparoscopic pyeloplasty. J Endourol. 2014;28(4):393-8.

40. Zhang J, Liu C. A training model for laparoscopic dismembered pyeloplasty. Nan Fang Yi Ke Da Xue Xue Bao. 2013;33(10):1541-3.

41. Díaz-Güemes Martín-Portugués I, Hernández-Hurtado L, Usón-Casaús J, Sánchez-Hurtado MA, SánchezMargallo FM. Ureteral obstruction swine model through laparoscopy and single port for training on laparoscopic pyeloplasty. Int J Med Sci. 2013;10(8):1047-52.

42. Jiang C, Liu M, Chen J, Wang P, Lin $\mathrm{T}, \mathrm{Xu} \mathrm{K}$ et al. Construct validity of the chicken crop model in the simulation of laparoscopic pyeloplasty. J Endourol. 2013;27(8):1032-6.

43. Raza SJ, Soomroo KQ, Ather MH. "Latex glove" laparoscopic pyeloplasty model: A novel method for simulated training. Urol J. 2011;8(4):283-6.

44. Valero RJ, Moanack J, Cruz G, Sánchez-Ismayel A, Sánchez-Salas R, García-Seguí A. Animal model for training in laparoscopic pyeloplasty. Actas Urol Esp. 2012;36(1):54-9.

45. Torricelli FC, Guglielmetti G, Duarte RJ, Srougi M. Laparoscopic skill laboratory in urological surgery: tools and methods for resident training. Int Braz J Urol. 2011;37 (1):108-11.

46. Yang B, Zhang ZS, Xiao L, Wang LH, Xu CL, Sun YH. A novel training model for retroperitoneal laparoscopic dismembered pyeloplasty. J Endourol. 2010;24(8):1345-9.

47. Teber D, Guven S, Yaycioglu O, Ugurlu O, Sanli O, Gozen AS, et al. Single-knot running suture anastomosis (one-knot pyeloplasty) for laparoscopic dismembered pyeloplasty: Training model on a porcine bladder 
and clinical results. Int Urol Nephrol. 2010;42(3):609-14.

48. Passerotti CC, Passerotti AM, Dall'Oglio MF, Leite KR, Nunes RL, Srougi M, et al. Comparing the quality of the suture anastomosis and the learning curves associated with performing open, freehand, and robotic-assisted laparoscopic pyeloplasty in a swine animal model. J Am Coll Surg. 2009;208(4):576-86.

49. Abraham JB, Abdelshehid CS, Lee HJ, Alipanah R, Andrade LA, Sargent ER, et al. LapED 4-In-1 silicone training aid for practicing laparoscopic skills and tasks: A preliminary evaluation. J Endourol. 2008;22(6):1351-7.

50. Fu B, Zhang X, Lang B, Xu $\mathrm{K}$, Zhang J, Ma $X$, et al. New model for training in laparoscopic dismembered ureteropyeloplasty. J Endourol. 2007;21(11):1381-5.

51. Ooi J, Lawrentschuk N, Murphy DL. Training model for open or laparoscopic pyeloplasty. J Endourol. 2006;20(2):149-52.

\section{Anexo}

Artículos publicados sobre modelos de entrenamiento para pieloplastia laparoscópica

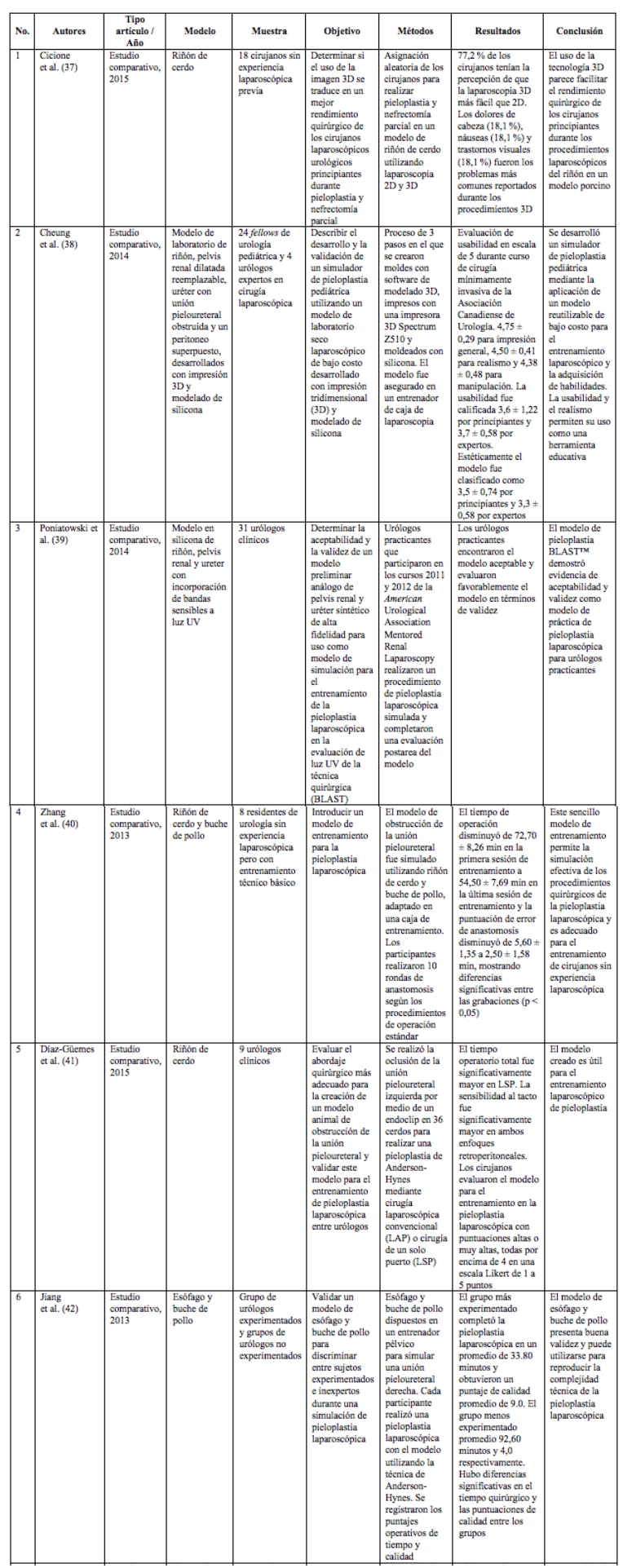


Pedro Villamizar, Marco Ángel, Valentina Corchuelo, et al.
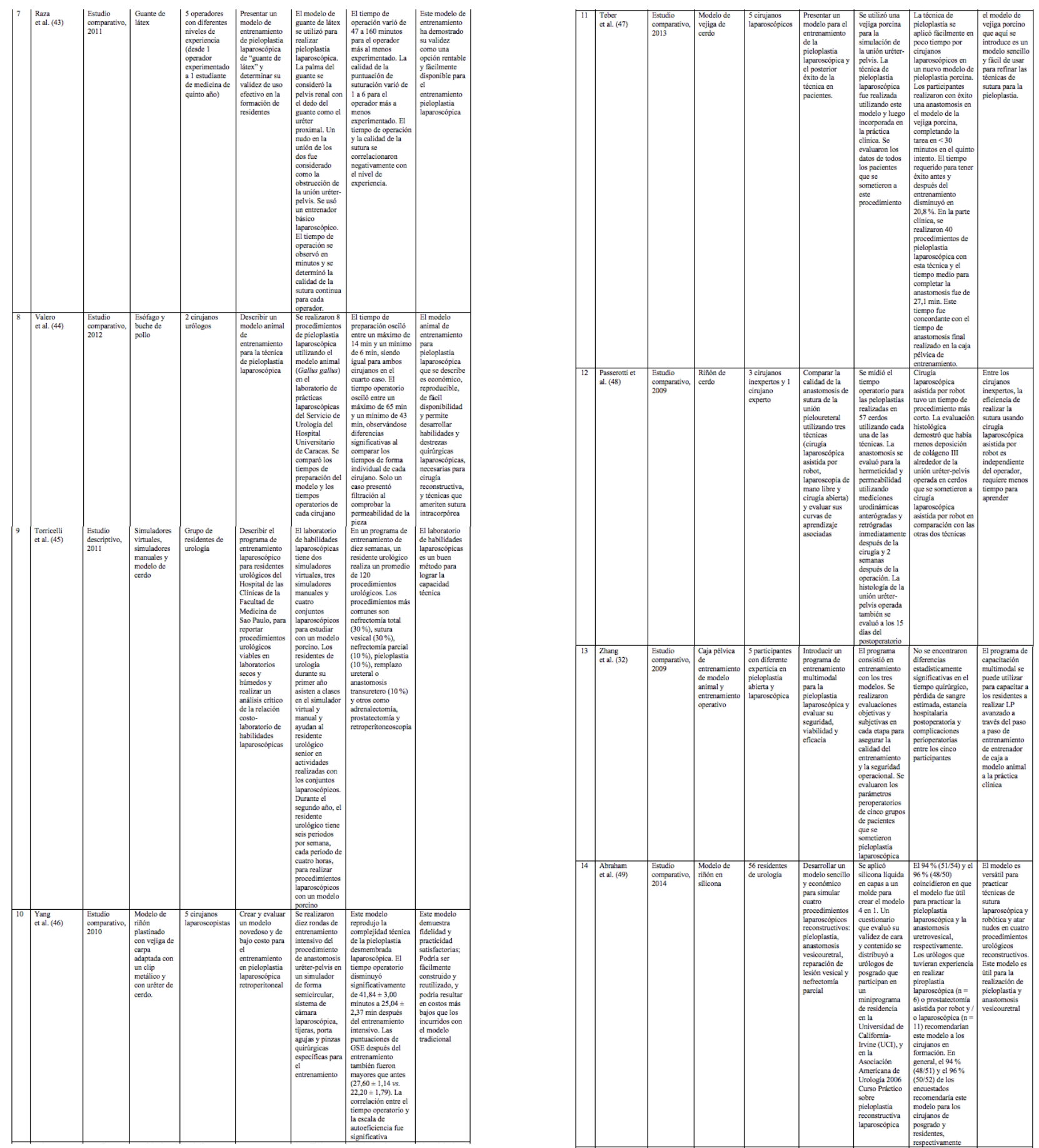


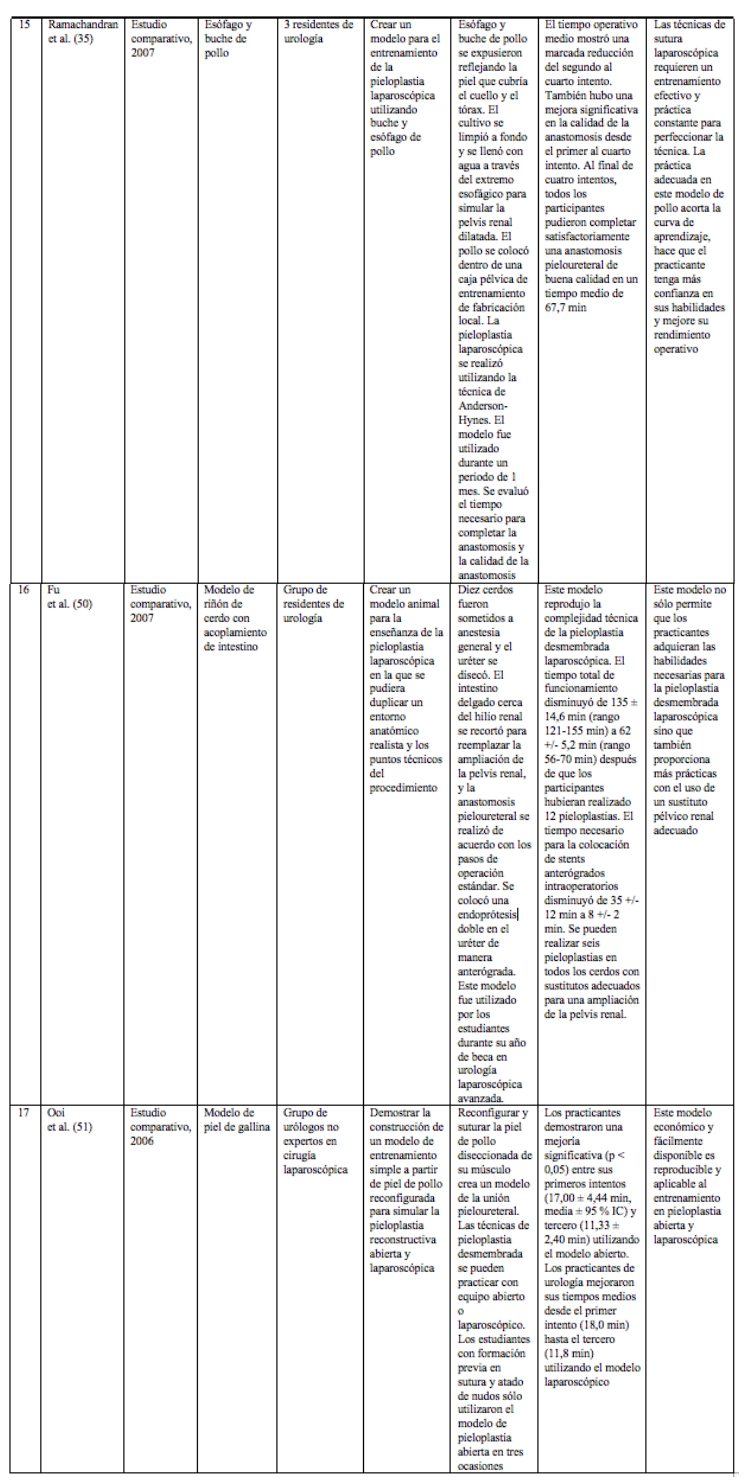

\section{Notas}

* Esta revisión de la literatura deriva de la propuesta "Integración de tecnologías de fabricación digital para el diseño y desarrollo de modelos anatómicos de entrenamiento en cirugía mínimamente invasiva. Caso: pieloplastia laparoscópica pediátrica", la cual fue financiada a través de la Convocatoria de Innovación 2017 de la Pontificia Universidad Javeriana (Cali, Colombia). 